X.

Aus der Psychiatrischen und Nervenklinik Strassburg. (Direktor: Geh. Med.-Rat Prof. Dr. Wollenberg.)

\title{
Zur pathologischen Anatomie und Pathogenese der juvenilen Paralyse.
}

\author{
Von \\ Dr. Scharnke, \\ Oberarat in 5. Westfäl. Inf.-Rgt. Nr. 53, kommandiert zur Klinik.
}

(Mit 1 Textligur.)

In Nr. 44 der Medizinischen Klinik, Jahrganz 1912, konnte Professor Rosenfeld über einen Fall von juveniler Paralyse aus der Strassburger Psychiatrischen und Nerrenklinik berichten, der die klinischen Eigentümlichkeiten der juvenilen Paralyse, nämlich die in der ersten Zeit ganz auffällig prävalierenden körperlichen Symptome, besonders deutlich erkennen liess. Es bestanden nämlich schon im ersten Krankheitsjahr neben starken Kopfschmerzen und häufigem Erbrechen ganz hochgradige Gang- und Gleichgewichtsstörungen und sehr starke Anarthrie, während sich psychisch nur eine stumpfe, langsam fortschreitende Demenz zeigte. Es wurde nun der Versuch gemacht, die schweren subjektiven und objektiven Hirnsymptome durch eine Dekompressivtrepanation (nach Cushing in der Gegend des r. Schläfenbeins) zu beseitigen. Tatsächlich verschwanden zunächst die schweren subjektiven Beschwerden, und auch die Gangstörung und der Tremor gingen wieder sehr zurück. Aber es war natürlich kein Dauerresultat, die Besserung hielt nicht lange an: Der Kranke ist dann allmäblich immer mehr verfallen; am Eude war er ein elendes Körperchen im Zustand äusserster Abmagerung und Blässe mit zahlreichen Dekubitalgeschwüren, mehrfachen unlösbaren Kontraktoren und anscheinend völligem Verlust jeglicher psychischen Funktion. Er reagierte auf keinen einzigen äusseren Reiz mehr, nur die Schmerzempfindlichkeit schien noch vorhanden zu sein. Im übrigen lag er völlig bewegungs- und teilnahmslos im Bett, wimmerte stundenlang leise vor sich hin und lebte nur den rein vegetativen Funktionen. Der Tod trat 
etwa 3 Jahre nach dem Manifestwerden der Krankheitssymptome ein, im Zustand des äussersten Marasmus.

Die Sektion konnte äusserer Umstände halber leider erst nach 38 Stunden vorgenommen werden, so dass manche wünschenswerten Untersuchungen unterbleiben mussten bzw. in ihren Ergebnissen nicht ganz einwandfrei blieben und somit nicht verwertet werden können. Insbesondere konnte nach so langer Zeit auf Spirochätennachweis nicht mehr gerechnet werden. Immerhin ergaben sich sehr interessante Befunde.

Nach den inzwischen bekannt gewordenen Untersuchungen besonders Sträusslers über die pathologisch-anatomischen Befunde juveniler Paralytiker und die besondere Rolle, die das Kleinhirn dabei spielt, dürfte es vielleicht von Interesse sein, die wichtigsten Ergebnisse der mikro- und makroskopischen Untersuchung kennen zu lernen.

Aus dem Sektionsprotokoll vom 13. Mai 1913 (Sekant Dr. Tilp) sei nur das Folgende hervorgehoben: „Schädel symmetrisch geformt, $49 \mathrm{~cm}$ im H. U. messend, in seinen vorderen und seitlichen Partien deutlich dicker, bis $6 \mathrm{~mm}$ dick. In der rechten Schläfengegend ein kreisrundes Loch von $1 \frac{1}{2} \mathrm{~cm}$ Durchmesser mit unregelmässigem Rande. Duralsack schlaff. Im Subduralraum reichlicher klarer Liquor angesammelt. An der Innenfläche der Dura eine leicht abziehbare gelbliche Membran aufgelagert, die insbesondere in der vorderen und mittleren Schädelgrube, sowie über der Konvexität der rechten Grosshirnhemisphäre ausgebildet ist. Die inneren Meningen blutreich, an der Konvexität gleichmässig verdickt. Die Arterien an der Hirnbasis zeigen normales Verhalten. Das Gehirn füllt die Schädelkapsel nicht aus, sondern ist deutlich kleiner. Das Totalgewicht beträgt $785 \mathrm{~g}$, die rechte Gehirnhemisphäre wiegt $305 \mathrm{~g}$, die linke $300 \mathrm{~g}$, das Kleinhirn mit Hirnstamm $135 \mathrm{~g}$. Die Ventrikel sind etwas weiter. Das Ependym in den Seitenventrikeln wenig, im 3 . und 4 . Ventrikel deutlicher, aber ganz fein granuliert. Auffällig ist, dass das Kleinhirn und auch der Hirnstamm von der Atrophie nicht befallen ist.

Das Relief der Scbädelbasis ist besonders nach vorne zu plump, insbesondere erscheint die Crista galli dick, die Keilbeinflügel abgerundet und plump. (Siehe die Figur.)

Die Sektion des Rückenmarks ergibt diffuse Verdickung der Leptomeningen."

Zusammenfassend also: „Atrophia cerebri, Hyperostosis cranii, Pachymeningitis interna et Leptomeningitis chronica. Defectus operativus cranii post trepanationem." Die letzte Todesursache hatte eine lobuläre Pneumonie abgegeben.

Sehr auffällig erschien die sehr starke Verdickung besonders der 
weichen Meningen. Diese produktive Leptomeningitis war am stärksten an der Konvexität ausgebildet, besonders in der Frontalgegend, ferner an der Fissura Sylvii. Hier kam es zur Bildung dicker Gewebsmassen von milchiger Trübung. Diese ausserordentlich starke Verdickung der Meningen fand sich vielfach auch an der Medulla, besonders im Bereich des Lumbalmarks.

Das Gehirn erschien also im ganzen stark atrophisch, insbesondere das Grosshirn. Dagegen liess sich, wie erwähnt, die gerade bei der

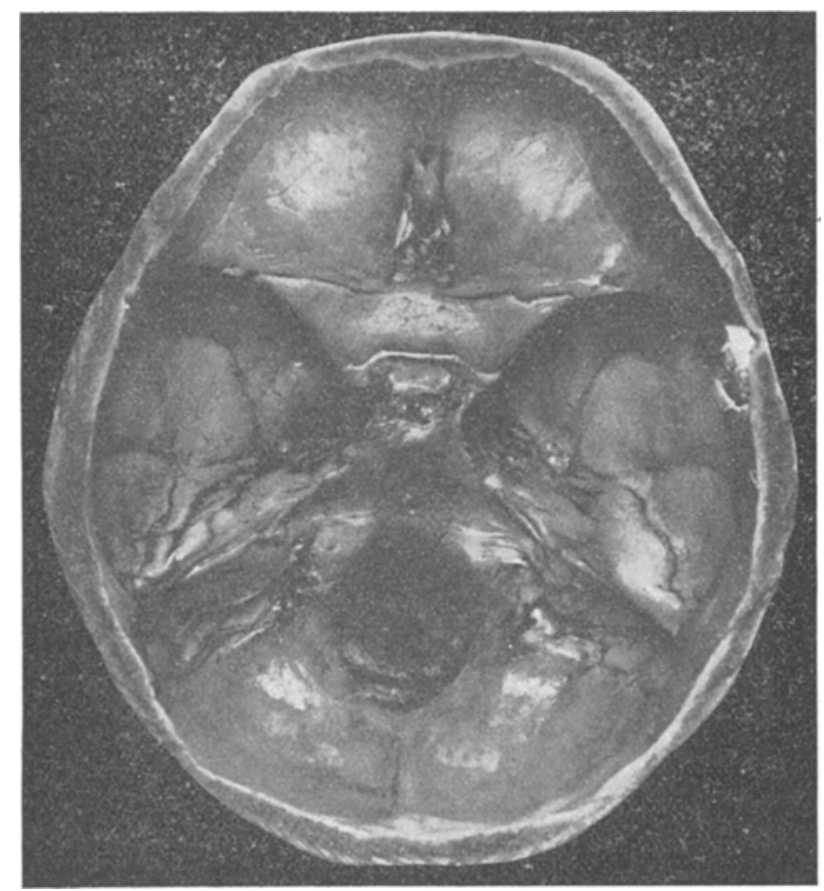

juvenilen Paralyse mehrfach beschriebene und von Sträussler zur zerebellären Heredoataxie in Beziehung gebrachte Kleinhirnatrophie und die Kleinheit der Medulla oblongata nicht nachweisen. Um so schöner sind die mikroskopischen Kleinhirnveränderungen ausgebildet. Die Atrophie des Grosshirns bezieht sich ganz besonders auf die Okzipitalgegend. In der Frontalgegend sind die basalen Windungen viel stärker atrophisch als die der Konvexität. Am geringsten mitgenommen scheinen die Temporallappen zu sein. Auch das Zwischenhirn und das Mittelhirn erscheinen relativ gut erhalten. Wir sehen also, dass die Beteiligung der 
Meningen und die Atrophie der Hirnabschnitte eine lokale Korrespondenz nicht erkennen lassen.

Eine Entwicklungsstörung glauben wir am Boden der Rautengrube nachweisen zu können. Hier verläuft die mediane, von der Schleifenkreuzung gebildete Raphe nicht in die Spitze der medianen Furche der Rautengrube hinein, vielmehr liegt diese Furche deutlich seitlich von der Raphe. Aehnliche Asymmetrien, besonders eine sehr erbebliche Ungleichheit des rechten und linken Hinterstrangs an absoluter Masse scheinen mehrfach im Verlauf der Medulla vorzuliegen, doch möchten wir hier nicht mit Sicherheit von Missbildungen reden, da gar zu leicht derartige Asymmetrien durch Schiefheit des Schnitts, eventuell auch durch ungleiches Vorgeschrittensein der Atrophien vorgetäuscht werden können, wiewohl die Asymmetrie vielfach überaus auffällig ist.

Die bei der Paralyse so häufige Ependymitis granularis fand sich schön ausgeprägt im 4, weniger stark auch in den Seitenventrikeln. Alle Ventrikel erschienen mässig erweitert, besonders auch die Ventrikelhörner.

Am Rückenmark liess sich schon makroskopisch besonders am gefärbten Präparat die Atrophie der Pyramidenbahnen sehr gut erkennen, entsprechend dem klinischen Befund (Spasmen, Reflexsteigerung, Babinski), wogegen die Hinterstränge relativ wenig mitgenommen zu sein scheinen.

Bekanntlich konnte nun Alzheimer 1912 in den "Ergebnissen auf dem Gebiet der pathologischen Histologie der Geistesstörungen" (Zeitschr. f. d. ges. Neurologie und Psychiatrie 1912, Bd. 5, H. 8) als gesichert annehmen, dass die auf hereditär-luetischer Grundlage entstandene Paralyse auffällig häufig mit eigentümlichen Veränderungen im Kleinhirn vergesellschaftet erscheint, besondess mit abnormer Bildung und Mehrkernigkeit der Purkinje'schen Zellen. Wir verdanken diese Befunde besonders Sträussler, der bereits 1910 die Ansicht aussprach, dass die auf der Grundlage einer hereditären Syphilis zur Entwickelung gelangenden Paralysen regelmässig im Zentralnervensystem Entwickelungsstörungen aufweisen, welche vornehmlich im Kleinhirn lokalisiert sind. Bevor wir diese Eigentümlichkeiten besprechen, die tatsächlich als sehr charakteristisch, wenn auch nicht als pathognomonisch für juvenile Paralyse erscheinen, seien die vorgefundenen mikroskopischen Grosshirnveränderungen besprochen:

Die Meningen lassen deutlich erkennen, dass ihre zum Teil sehr starke Verdickung nicht etwa nur durch Gefässwucherung und Plasmazelleninfiltration zustande gekommen ist, vielmehr finden sich vielfach dicke Balken neuen festen Bindegewebes, zum Teil noch mit Fibro- 
blasten. Diese neugebildeten Bindegewebsmassen Jassen sich an der Leptomeninx der Medulla zum Teil ziemlich oder ganz scharf von einer Verdickung der gliösen Randmembran trennen, die besonders im Bereich der Seitenstränge vielfach sehr deutlich sich nachweisen lässt.

An den Ganglienzellen fällt an vielen Stellen die scheinbare Regellosigkeit ihrer Lagerung auf. Nicht nur erscheint die Trennung der Schichten verwischt, sondern die Pyramidenzellen z. B. liegen vielfach garnicht mehr mit der Spitze nach der Rinde hin orientiert, sondern wie auf die Seite gelegt. Sie sind meist auch nicht mehr gleichmässig verteilt, sondern liegen hier dichter, dort vereinzelter. An vielen Stellen ist die Rinde ganz auffällig stark verschmälert, die Molekularschicht z. B. fast verschwunden. Es wird sich nicht entscheiden lassen, ob das angeborene Anomalien sind oder aber ob diese Architekturstörung erst im Laufe des Krankheitsprozesses entstanden ist, ob also da, wo die Zellen dichter liegen, mehr Zwischensubstanz zugrunde gegangen ist oder ob da, wo die Zellen spärlicher liegen, mehr Zellen verschwunden sind. Da aber die erwähnte Störung der gleichmässigen Zellenverteilung und die Störung des Schichtenaufbaues vielfach so ungemein stark ist, so neige ich doch der Meinung zu, es müsse sich hier wenigstens teilweise um Störungen der Entwicklung handeln. Sehr auffällig ist auch die vielfach sehr verschiedene Grösse der Ganglienzellen. Ganz besonders gross sind meistens die der Verkalkung anheimgefallenen Zellen. Die Protoplasmaveränderung ist bei allen Pyramidenzellen überaus hochgradig. Ab und zu sieht man sehr schön die wabige Veränderung. Vielfach erscheint das Protoplasma an Masse sehr reduziert, oft fast oder ganz fehlend, so dass der Kern isolirt zu liegen scheint. Die Tigroidzeichnung ist nirgends mehr erhalten. Sehr schön ist vielfach die Fältelung der Kernmembran nachweisbar. Leider lassen sich diese Veränderungen nur zum Teil auf den Krankheitsprozess als solchen mit Sicherheit beziehen, zum Teil fallen sie wohl auch Fäulnisvorgängen wegen der verspäteten Sektion zur Last.

Ganz besonders schön stellt sich an Weigert'schen Markscheidenpräparaten der besonders von Tuzek beschriebene Markfaserschwund dar. Er ist stellenweise derartig hochgradig, dass die Markleiste in der Windung völlig zu fehlen scheint. Auch die Tangentialfaserschicht ist an vielen Stellen ausserordentlich stark gelichtet.

Ganz auffällig wenig finden sich Mastzellen und ebenso vermisst man die Stäbchenzellen fast völlig. Ich habe in den zahlreichen durchmusterten Schnitten nicht eine einzige einwandfreie Stäbchenzelle gefunden. Es ist wenig wahrscheinlich, dass die sonst bei der Paralyse so zahlreich gefundenen Zellen ebenfalls der verspäteten Sektion zum 
Opfer gefallen sind, so dass sie überhaupt nicht mehr nachweisbar waren. Jedenfalls erscheint uns ihr Fehlen sehr auffällig.

Plasmazellen, die für die Paralyse am meisten charakteristischen Zellen, finden sich sehr zahlreich, sowohl in den Hirngefässen selbst als auch in den Meningen. Ausserhalb der Gefässe, weiter getrennt von ihnen, lassen sie sich aber nur bie und da nachweisen, besonders im Kleinhirn. Ueber ibre Beziehungen zu den Abbauprodukten s. weiter unten.

Diese sogen. Abbauprodukte finden sich in ganz ungeheuren Massen, so dass man schon daraus die juvenile Paralyse als destruierenden Prozess schwerster Art bezeichnen muss. Es lässt sich an vielen Stellen zeigen, dass da, wo die Ganglienzellen den stärksten Verfall zeigen und vielfach kaum mehr Protoplasma aufweisen, die Menge der Abbauprodukie am grössten ist. In diesen Gegenden erscheinen auch die Blutgefässe vielfach stark geschlängelt, so dass ihr Lumen in einem Schnitt mehrfach getroffen ist. Die Abbauprodukte selbst sind vielfach überaus grobkörnig, erfüllen die Adventitialscheiden der Gefässe mit dicken körnigen Massen, meist kugelig, selten eckig. Bisweilen erscheinen diese Massen noch deutlich in Zellen eingelagert, ähnlich den Fettklümpchen in den Körnchenzellen.

Die Blutgefässe selbst zeigen ansser der schon erwähnten häufig sehr starken Schlängelung an vielen Stellen eine starke lymphozytäre Infiltration, besonders der Scheiden, abgesehen von den zahlreich vorhandenen Plasmazellen. Für das herdförmige Auftreten der adventitiellen Zellinfiltration lässt sich aus dem histologischen Bild irgend eine Erklärung nicht ableiten. Häufig erscheinen die Kapillaren abnorm zahlreich, besonders in der Medulla, als ob das zwischen ihnen liegende Gewebe stark atrophiert wäre. Endarteriitische und Gefässobliterationserscheinungen finden sich nur relativ wenig.

Was nun den Sträussler'schen, inzwischen noch mehrfach bestätigten Befund der doppelkernigen Purkinje'schen Zellen im Kleinhirn betrifft, so finden sich diese Zellen in unserem Fall in grosser Zahl. Die Bilder sind zum Teil so deutlich, dass sie sich photographisch gut festbalten lassen. Die Zellen finden sich in jedem Schnitt, meist mehrfach. Wenn sie sich auch vereinzelt bei anderen Psychosen finden, so dürften sie in solcher Menge doch wohl ein Charakteristikum der juvenilen Paralyse darstellen.

Noch viel eindrucksvoller aber als diese Doppelkernigkeit der Zellen erscheint unter dem Mikroskop die hochgradige Störung der Lagerung der Purkinje'schen Zellen: während sie an manchen Stellen ausgerichtet, wie Soldaten, an der Grenze von grauer und granulierter 
Schicht sich finden, mit immer gleichen Abständen, sind an anderen viel zahlreicheren Stellen diese Abstände überaus nngleich. An vielen Stellen fehlen die Purkinje'schen Zellen auf lange, fast eine. Windung einnehmende Strecken ganz, vereinzelt liegen sie auch abnorm dicht. Endlich sind sie vielfach ganz falsch gegen die Kleinhirnoberfläche orientiert, liegen wie umgeworfene Bäumchen da, so dass der Dendrit parallel zur Zellschicht liegt, statt senkrecht drauf zu stehen. Ab und $\mathrm{zu}$ erscheinen einzelne Zellen völlig in die umgebenden Schichten versprengt, geringere Verschiebungen sind fast die Regel. Wie bei den Pyramidenzellen im Grosshirn finden sich auch hier bei den Purkinjeschen Zellen vielfach ganz auffällige Grössenunterschiede. Alles in Allem also weisen die Purkinje'schen Zellen ganz besonders hochgradige Störungen in ihrer Entwicklung, Verteilnng und speziellen Lagerung auf.

Die Ansicht Sträussler's, dass sich die angeborenen Veränderungen bei der juvenilen Paralyse ganz besonders im cerebello-spinalen System finden, bestätigten uns ferner einige sehr interessante, im Rückenmark vorgefundene Veränderungen. Fast in jedem Schnitt nämlich findet mau Ganglienzellen, die völlig aus der grauen Substanz versprengt, mitten in den Vorder-, Seiten- oder Hintersträngen liegen. Die relative Häufigkeit dieses Befundes lässt es als ausgeschlossen erscheinen, dass diese Zellen etwa durch das Mikrotom verschleppt sein könnten. Beweisend dafür, dass diese Zellen auch im Leben schon so heterotopisch gelegen haben, dürfte wohl auch der Umstand sein, dass sie stets in Bindegewebssepten liegen und dass man zum Teil noch ihre Fortsätze deutlich in ihrem Verlauf verfolgen kann.

Endlich liess sich mikroskopisch in mehreren Schnitten nachweisen, dass der Zentralkanal mehrfach eine seitliche Abschnürung, eine unvollkommene Verdoppelung zeigte.

Nach dem bisher Geschilderten bot sich also sowohl im Grosshirn als auch im Kleinbirn und in der Medulla ein sehr charakteristischer Befund dar. Dagegen ist uns der Nachweis der Pallida trotz vielfacher Versuche nicht gelungen, weder mit der Levaditi'schen noch mit der Noguchi'schen Färbung. Wohl fanden wir vielfach pallidaähnliche geschraubte Gebilde, aber wir mussten sie stets als mitgefärbte Nervenfasern ansprechen. Auffällig waren uns aber vielfach dichte Ansammlungen kleinster Körnchen, aus denen man sich ohne viel Phantasie Spirochäten zusammengesetzt hätte denken können. Es muss natürlich als völlig ungewiss dahingestellt bleiben, ob es sich dabei etwa tatsächlich um zerfallene Spirochäten gehandelt hat. Schliesslich ist es ja auch wegen der verspäteten Sektion begreiflich, dass wir keine Spirochäten mehr fanden. 
Die oben erwähnten Befunde, besonders die so stark verlagerten Ganglienzellen und die Verdoppelnng des Zentralkanals, dürften wohl ebenso wie die architektonischen Störungen in der Grosshirnrinde und in der Lagerung der Purkinje'schen Zellen in der Tat nicht anders als durch eine Keimschädigung zu erklären sein, und es bleibt die Frage offen, ob nur der schon im Keim familiär belastete Fötus, wenn er vom Vater oder von der Mutter her dazu noch syphilitisch wird, später paralytisch wird, ob also die syphilitische Infektion zu einer anderen Keimanomalie hinzukommen muss, um eine juvenile Paralyse hervorzubringen. Nach allem, was bisher an mikroskopischen Befunden bekannt geworden ist, besonders nach den Untersuchungen Sträussler's, könnte man fast zu der Ansicht kommen, dass nur solche Kinder an juveniler Paralyse erkranken, die ohne die Lues einer der anderen hereditären Erkrankungen, insbesondere des cerebellospinalen Systems zum Opfer gefallen wären oder die sonst Zeichen eines in irgend einer Richtung besonders binfälligen Zentralnervensystems geboten hätten. Auch hier erbebt sich eben wie bei den erwachsenen Paralytikern die Frage: Warum werden von so vielen kongenital-syphilitischen Kindern nur so wenige juvenil-paralytisch.

Einige besondere Betrachtungen möchte ich noch an die Kleinhirnbefunde anknüpfen: Die starke Veränderung gerade der Purkinje'scben Zellen erscheint mir ganz besonders bemerkenswert: sie steht im lebhaftesten Gegensatz zum makroskopischen Befund. Wie eingangs erwähnt, schien ja gerade das Kleinhirn gar nicht atropisch, überhaupt kaum von dem paralytischen Prozess betroffen, und auch mikroskopisch finden sich keine sehr erheblichen, insbesondere nur wenig entzündliche Veränderungen. Nur die funktionell vermutlich wohl am böchsten stehenden, am kompliziertesten gebauten Purkinje'schen Zellen sind so stark betroffen. Da scheint mir im Verein mit den anderen Missbildungen doch die Annahme sehr nahe zu legen, dass es sich hierbei nicht um etwas erst intra vitam Gewordenes handelt, sondern um eine schon in der Entwicklung des Fötus entstandene Störung, also um eine angeborene Missbildung. Stebt diese nun nur zur Lues in Beziehung, ist sie die Folge der elterlichen Syphilis? Bekanntlich haben nun neuere Autoren das Vorkommen doppelkerniger Purkinje'scher Zellen auch bei anderen Psychosen beschrieben, insbesondere will ich hier eine Arbeit von Stein anführen (Zeitschrift für die gesamte Neurologie und Psychiatrie Bd. 21, Heft 5). Derselbe fand diese doppelkernigen Zellen in $84 \mathrm{pCt}$. bei den erwachsenen Paralytikem und in einem allerdings weniger bohen Prozentsatz auch bei anderen Geisteskranken, während sich bei 15 Geistesgesunden keine einzige solche Zelle 
fand. Wie ist das zu erklären? Es scheint mir das doch ein weiteres Zeichen dafür zu sein, dass nur ein von vornherein dazu prädisponiertes, also in einer bestimmten Richtung minderwertiges Gehirn paralytisch wird, wenn es von der luetischen Infektion betroffen wird. Die angeborene Minderwertigkeit dokumentiert sich unter anderem eben auch durch das Vorkommen solcher Zellen. Von ganz besonderem Interesse ist es deshalb, dass einerseits Stein, wie erwähnt, in 15 normalen Kleinhirnen keine einzige doppelkernige Purkinje'sche Zelle fand, andererseits aber bei 3 Hirntumoren in allen Fällen, und gerade bei den Hirntumoren nimmt man doch im allgemeinen an, dass sie auf Basis einer Anlageanomalie zur Entwicklung kommen (Stein). Auch Stein kommt daher zu der sehr interessanten Ansicht: "Wenn die doppelkernigen Ganglienzellen nur intrauterin entstehen würden, dann müssten die bier mitgeteilten Befunde zu der Auffassung führen, dass die Mehrkernigkeit ein anatomisches Merkmal einer zu Psychosen führenden Anlage darstellt." Dass sie intrauterin entstehen, bleibt freilich noch $z u$ beweisen.

Eine weitere Stütze erhält diese Ansicht durch die interessante Arbeit von Sibelius. (Rückenmarksanomalien bei Paralytikern, Ziegler's Beiträge 51, 1911.) Derselbe untersuchte 24 paralytische und 15 normale Rückenmarke auf innere Degenerationszeichen (architektonische Missbildungen, zweikernige Ganglienzellen, fehlende Pyramidenvorderstränge u. dgl.), und es ergab sich, dass jedes normale Rückenmark 0,93, jedes paralytische 2,71 Anomalien aufweist.

An dieser Stelle darf icb vielleicht eine kleine Beobachtung einfügen, die ich selbst an den zahlreichen Paralytikern unserer Klinik gemacht habe, die aber von mir an grösserem Naterial noch nachzuprüfen sein wird. Ein ganz auffallend hoher Prozentsatz der Paralytiker zeigt ein ganz eigenartiges, fast charakteristisches äusseres Ohr. Das Ohrläppchen ist dabei gut ausgebildet, aber in seiner ganzen Ausdehnung mit der Wange verwachsen und nicht selten sogar in eine in die Wange auslaufende Falte ausgezogen. Ich fand diese Art der Ohrenläppchenbildung z. B. unter den 14 letzten genauer beobachteten Paralytikern $9 \mathrm{mal}$. Viel seltener findet sich das verkümmerte rudimentäre und ebenfalls ganz verwachsene Ohrläppchen, wie es bei anderen Psychosen und natürlich auch bei Geistesgesunden häufig vorkommt. Ohne dass ich der Eigenart des Ohrläppchens eine irgendwie grosse. Bedeutung beilege, schien es mir doch nicht angezeigt, diese Beobachtung ganz zu übergehen, denn sie scheint mir vielleicht zu den von Sträussler, Sibelius und Stein gemachten Beobachtungen gut zu passen.

Nach allem bisher Gesagten kann ich mich nur der erst jüngst von dem hervorragenden Paralysekenner Pilcz geäusserten Ansicht 
anschliessen (Med. Klink 1914, Nr. 19), dass es eine spezifische Veranlagung zur Paralyse gibt, die quantitativ und qualitativ anders geartet ist, als die bekannte hereditär-degenerative.

Es gibt ja auch noch andere klinische Erfahrungstatsachen, die für die Annahme einer spezifischen Paralysedisposition sprechen. Wie schon Pilcz sagt, ist es sicher sehr auffallend, wie selten Psychopathen, Dégénérés, Verbrechernaturen an Paralyse erkranken, obwobl doch gerade diese Art von Menschen besonders häufig sich als syphilitisch erweist. Ferner sind doch Kombinationen von Paralyse etwa mit manischdepressivem Irresein, Dementia praecox und Epilepsie ganz ungemein selten. Und auf der anderen Seite wirkt die Paralyse gerade deshalb so tragisch, weil wiederum erfahrungsgemäss meist sonst körperlich und geistig gesunde, im Leben brauchbare, sozial tüchtige Elemente von ibr befallen werden. Die Annahme einer besonderen Paralysedisposition, die in einem gewissen Antagonismus zur degenerativen Veranlagung steht, hat somit viel Bestechendes an sich. Diese Annahme einer ganz bestimmt gearteten Disposition zur Paralyse steht nicht im Gegensatz zu der oben ausführlich dargestellten Anschauung, dass zur Entwicklung der Paralyse innere Degenerationszeichen des Zentralnervensystems gehören. Denn das Vorhandensein von Degenerationszeichen wie Doppelkernigkeit usw. spricht selbstverständlich gar nicht gegen die soziale Brauchbarkeit und körperliche Tüchtigkeit der Träger und steht in gar keinem Zusammenhang mit der erblich degenerativen psychopathischen Konstitution, deren anatomisches Substrat wir überhaupt nicht kennen und vielleicht niemals kennen werden.

Freilich wird mit der Annahme einer solchen Veranlagung das Zustandekommen der Paralyse noch immer vicht erklärt, aber vielleicht dem Verständnisse etwas näher gerückt. Dass überhaupt eine besondere Disposition bestimmt gearteter Individuen einer bestimmten Infektion gegenüber denkbar ist, dafür sprechen auch die Steiner'schen Impfversuche: Bei albinotischen Kaninchen lassen sich syphilitische Erscheinungen im Zentralnervensystem viel leichter und in höherem Prozentsatz erzielen als bei anderen Kaninchen. Nach all dem bisher Gesagten scheint mir Obersteiner das Richtige getroffen zu haben, wenn er sagt: Paralyticus nascitur atque fit.

Zum Schlusse erwächst mir die angenehme Pfilcht, Herrn Professor Chi a ri für die Ueberlassung des Sektionsprotokolls und Herrn Dr. Steiner für die Unterstützung bei den histologischen Untersuchungen meinen herzlichen Dank auszusprechen. Ganz besonderen Dank schulde ich Herrn Professor Wollenberg für die Ueberiassung des Materials. 
Zur pathologischen Anatomio und Pathogenese der juvenilen Paralyse. 313

\section{Literaturverzeichnis.}

1. Alzheimer, Ergebnisse auf dem Gebiet der pathologischen Histologie der Geistesstörungen. Zeitschr. f. d. ges. Neurol. u. Psych. 1912. Bd. 5. Heft 8.

2. Obersteiner, Ueber pathologische Veranlagung am Zentralnervensystem. Wiener klin. Wochenschr. 1913. Nr. 14.

3. Pilcz, Die jüngsten Fortschritte auf dem Gebiet der Lehre von der progressiven Paralyse. Mediz. Klinik. 1914. Nr. 19.

4. Rosenfeld, Ueber juvenile Paralyse. Ebendas. 1917. Nr. 44.

5. Sibelius, Rückenmarksanomalien bei Paralytikern. Ziegler's Beiträge. 1911. 51.

6. Stein, Die Bedeutung der mehrkernigen Ganglienzellen. Zeitsohr, f. d. ges. Neurol, u. Psych. Bd. 21. Heft 5.

7. Sträussler, Ueber Entwicklungsstörungen im Zentralnervensystem bei der juvenilen progressiven Paralyse ond die Beziehungen dieser Erkrankung za den hereditären Erkrankungen des Zentralnervensystems. Ebendas. 1910. Bd. 2. 